\title{
Editorial
}

\section{Carotid occlusion}

\author{
Roberto C. Heros, M.D.
}

\section{Department of Neurosurgery, University of Miami, Florida}

It is a pleasure to be given the opportunity to comment on the important paper by Grubb and colleagues, ${ }^{6}$ which appears in this issue of the Journal of Neurosurgery. As I prepare myself for this task by reviewing the report on the initial extracranial-intracranial (EC-IC) bypass study ${ }^{3}$ and its early critical reviews, ${ }^{1,2,4,8}$ as well as the original report on the Carotid Occlusion Surgery Study (COSS) ${ }^{7}$ and the earlier background report that laid its rationale, ${ }^{5} \mathrm{I}$ end up with a certain melancholy and heavy heart but also a tremendous amount of pride in our specialty. The bypass operation played a major role in stimulating me, and several other young neurosurgeons of my generation I am sure, to specialize in the field of cerebrovascular surgery. The ability to perform an anastomosis in vessels as small as 1 $\mathrm{mm}$ and see on an angiogram a few months later that this bypass can provide luxuriant flow to a previously underperfused middle cerebral artery (MCA) territory was nothing short of miraculous and undoubtedly made our senior mentors at the time think of those who began to do this operation while residents as technical whiz kids. No wonder then that we received the results of the initial EC-IC bypass study with considerable disappointment, and that we now find that even the group of patients we little doubted would benefit from this operation, in general, appears to receive very little benefit at the expense of relatively high morbidity. The pride I feel in reviewing this topic comes from the realization that neurosurgeons, in collaboration with our colleagues in the neurological community, enamored of the bypass operation as many of us are, have had the scientific fortitude to question its effectiveness and to do so in a most rigorous scientific manner with well-conducted unimpeachable clinical trials.

First, I will comment on some of the criticisms that I have heard about the COSS. The following paper by Grubb and associates goes a long way in answering what perhaps has been the most important criticism, and that is that the operations were performed by inexperienced surgeons, which accounted for the unacceptable perioperative morbidity. Perhaps the most important information provided in their paper is the list of neurosurgeons who performed the bypasses in the COSS. It would be hard for any neurovascular surgeon in North America to deny that almost all of the individuals on that list are recognized as experienced cerebrovascular surgeons and that many of them have not only performed many of these operations, but have also contributed to the literature of bypass surgery. Authors of the COSS opened themselves, perhaps unnecessarily, to this criticism by accepting among the criteria for surgical certification attendance at a training workshop held in St. Louis or by allowing provisional certification by performing an operation in the study under the supervision of the principal surgical investigator or a designee. Looking at the list of surgeons and knowing most of them personally, it is clear that they had experience with bypass surgery, although in recent years that experience may have been more with bypasses for unclippable aneurysms or carotid sacrifice for other reasons rather than for ischemic events. Perhaps, despite this experience, many of these surgeons could not provide complete records for 10 recent EC-IC bypasses performed specifically for ischemia, and hence the necessity to loosen the criteria for certification of these individuals. Even if one were to accept that several of the surgeons were relatively inexperienced with bypass grafts for ischemia, there are data in the paper by Grubb and colleagues that suggest that, in fact, inexperience of the operators was not a problem. The bypass patency rates of $98 \%$ at 30 days postoperatively and $96 \%$ at the last follow-up examination are perhaps the most important facts to counteract the claims of inexperience. The average time for occlusion as well as the maximum time-although perhaps not competitive with the expediency with which we used to perform these operations during those few years before the report on the original bypass study, ${ }^{3}$ when some of us were doing 40 or 50 of these operations per year-is still reasonable and within the range achieved today by even the most experienced operators.

A corollary of the inexperienced surgeons criticism is that the surgical morbidity was too high. Indeed it was. Even though the authors indicate that the $15 \%$ rate of perioperative ipsilateral hemispheric stroke in the COSS is not statistically different from the $12.2 \%$ rate in the initial ECIC study, the fact is that it is different and that maybe it is only because of the smaller group of patients in the COSS that the difference did not reach statistical significance. In addition, 12 of the 93 surgically treated patients in the COSS had serious perioperative morbidity, such as subdural hematoma, myocardial infarct, severe respiratory distress, and wound infection. Since I do not believe that surgical inexperience was a major factor, I am left to conclude that the high operative morbidity encountered in the COSS is related to the fact that this particular group of patients, selected for their presumed high risk for future stroke, is also at high risk for operative morbidity and perioperative 


\section{Editorial}

stroke not only because of their compromised neurological status, but also because the existence of other comorbidities is so frequent in these patients. Unfortunately, this is a fact that we are probably unable to change.

Investigators in the COSS believe that the main reason the study failed to show an advantage for the surgical group is the fact that the medical group fared so much better than expected (22.7\% rate of ipsilateral stroke over a 2-year period as opposed to the expected $40 \%$ ). This conclusion has led to another important criticism of the COSS, and that is that the methodology used to select this high-risk group of patients was inadequate and indeed failed to select such a group. This criticism has been based on the fact that the PET measurement methodology was slightly different in the COSS than in their initial background study that clearly selected a high-risk group of patients. ${ }^{5}$ I would rather not comment about this because I am not an expert in PET, but I suspect that the authors, perhaps in response to this editorial, will be able to provide evidence to the effect that the methodology used to select high-risk patients in the COSS study was comparable to that used in their previous background study. So, if indeed the group of patients in the COSS is similar to the previous group that they used as rationale for embarking on this study, then why did the medically treated group fare so much better in the COSS? Could it be that medical therapy has improved so much in less than a decade? Certainly, statins were used at the time of the original study, although perhaps not as widely as they are now. Perhaps the authors can comment further on this in their reply to this editorial, but I doubt that the improvements in medical therapy for the nonsurgically treated patients within the short period of time between the 2 studies can account for the fact that the medical group fared so much better than expected in the COSS.

Several of my cerebrovascular colleagues have commented that the study should have been allowed to go on, and that if it had, a significant advantage would have eventually been observed in the surgically treated group. Indeed, the Kaplan-Meier cumulative curves for analysis of the primary end point do cross at 2 years, and it is possible that in time the advantage of surgery may have reached statistical significance. Obviously, this was very much considered and discussed by experts on the data safety monitoring board that performed the futility analysis that led to termination of the trial. Their conclusion was that even if they had lowered the expectation for a significant difference to $10 \%$, the number of patients that needed to be recruited into the trial would have been 986 to achieve an $80 \%$ power. Knowing how difficult it was to recruit the 195 patients for the COSS, it is understandable that the study was terminated. Another very important point that at times we forget when we consider the "crossing of curves" is that the 2 curves have a very different slope, with much of the stroke morbidity in the surgical group occurring in the perioperative period, whereas in the medically treated group, it occurred gradually over the 2-year period. Even without performing a sophisticated life table analysis, anyone should understand that those patients who had a stroke in the perioperative period had to live with their incapacity from then on as opposed to the more gradual occurrence of stroke in the nonsurgical group.
Another legitimate criticism that has been leveled at the COSS is that patients entered into the study were relatively stable from a neurological point of view. Many of them had only a single mild stroke or transient ischemic attack (TIA) several weeks before being entered into the study, and in fact some were able to fly a considerable distance to go to another center to undergo their PET study. Clearly, these patients, who may have constituted the majority of patients in the COSS, are different from those who are incapacitated from multiple small strokes or TIAs that continue despite maximal medical therapy after carotid occlusion has been found. The problem, of course, is that if the surgical morbidity was high in the COSS group of relatively stable patients, wouldn't it have been higher in this theoretical group of unstable patients? We will never know the answer because it is impossible to find enough such unstable patients to be able to perform a randomized study. However, it should be remembered that in the initial EC-IC study, this particular group of patients who had persistent symptoms after demonstrating carotid occlusion was one of the two groups (the other group consisted of patients with MCA stenosis) that in the post hoc subgroup analysis fared worse with bypass surgery than with medical therapy. ${ }^{3}$

I will end on a personal note by commenting on what the COSS tells me. As a disclaimer, I will say that I have a strong bias against becoming enslaved by Level I evidence in my clinical decision making, although, clearly, strong Level I evidence, of which the COSS study is an excellent example, provides a powerful background factor influencing but not dictating such decisions. Should the results of the COSS determine that we will never again recommend an EC-IC bypass for cerebral ischemia, even in the group of patients that we can now identify as being at high risk for future stroke? In view of the results of this study, would I recommend a bypass to a patient who had a small stroke or TIA 2 months ago when he or she demonstrated an occlusion of the ipsilateral carotid artery and has been stable since, even though he or she now has physiological evidence of "misery perfusion" on PET, SPECT, or some other methodology? Clearly, I would not now recommend a bypass for such a patient, but I must admit that perhaps neither would I have done so before this study. On the other hand, what about a patient who has continued to have prolonged TIAs believed to be on a hemodynamic basis since he or she demonstrated carotid occlusion, has no medical comorbidities, has failed maximal medical therapy, and has evidence of misery perfusion? I believe that, despite results of the COSS study and the initial EC-IC study, I would today recommend a bypass to such a patient; however, I would have to make sure that this patient understood that there would be a significant risk of perioperative morbidity if he or she decided to proceed with surgery.

The neurological community should be very proud of the COSS. I heartily congratulate its leaders and the many contributors to this excellent effort.

(http://thejns.org/doi/abs/10.3171/2012.6.JNS121106)

\section{Disclosure}

The author reports no conflict of interest. 


\section{References}

1. Ausman JI, Diaz FG: Critique of the extracranial-intracranial bypass study. Surg Neurol 26:218-221, 1986

2. Day AL, Rhoton AL Jr, Little JR: The extracranial-intracranial bypass study. Surg Neurol 26:222-226, 1986

3. EC/IC Bypass Study Group: Failure of extracranial-intracranial arterial bypass to reduce the risk of ischemic stroke. $\mathbf{N}$ Engl J Med 313:1191-1200, 1985

4. Goldring S, Zervas N, Langfitt T: The Extracranial-Intracranial Bypass Study. A report of the committee appointed by the American Association of Neurological Surgeons to examine the study. N Engl J Med 316:817-820, 1987

5. Grubb RL Jr, Derdeyn CP, Fritsch SM, Carpenter DA, Yundt $\mathrm{KD}$, Videen TO, et al: Importance of hemodynamic factors in the prognosis of symptomatic carotid occlusion. JAMA 280: 1055-1060, 1998

6. Grubb RL Jr, Powers WJ, Clarke WR, Videen TO, Adams HP Jr, Derdeyn CP: Surgical results of the Carotid Occlusion Surgery Study. Clinical article. J Neurosurg [epub ahead of print October 26, 2012. DOI: 10.3171/2012.9.JNS12551]

7. Powers WF, Clarke WR, Grubb RL Jr, Videen TO, Adams HP Jr, Derdeyn CP: Extracranial-intracranial bypass surgery for stroke prevention in hemodynamic cerebral ischemia: the Carotid Occlusion Surgery Study Randomized Trial. JAMA 306:1983-1992, 2011

8. Sundt TM Jr: Was the international randomized trial of extracranial-intracranial arterial bypass representative of the population at risk? N Eng J Med 316:814-816, 1987

\section{Response}

Robert L. Grubb JR., M.D., ${ }^{1,2}$ William J. Powers, M.D., ${ }^{4}$ William R. Clarke, Ph.D., ${ }^{5}$ Harold P. Adams JR., M.D., 6 and Colin P. Derdeyn, M.D. ${ }^{1-3}$

Departments of ${ }^{1}$ Neurological Surgery, ${ }^{2}$ Radiology, and ${ }^{3}$ Neurology, Washington University School of Medicine, St. Louis, Missouri; ${ }^{4}$ Department of Neurology, University of North Carolina School of Medicine, Chapel Hill, North Carolina; ${ }^{5}$ Clinical Trials Statistics and Data Management Center, University of Iowa College of Public Health; and ${ }^{6}$ Department of Neurology, University of Iowa Carver School of Medicine, Iowa City, Iowa

We are grateful to Dr. Heros for his thoughtful remarks about the COSS and the role of EC-IC arterial bypass in patients with ischemic cerebrovascular disease. The COSS investigators were also disappointed with the results of the study, as we were confident before the study began that superficial temporal artery-middle cerebral artery (STA-MCA) bypass would benefit patients with symptomatic carotid artery occlusion and impaired cerebral hemodynamics. Findings in the COSS trial have led to a number of criticisms, with many being the same invalid criticisms raised about the initial EC-IC bypass trial. ${ }^{1,5,9,11,18}$ Dr. Heros has refuted several of these criticisms in his insightful editorial. Inexperienced surgeons performing the operations do not explain the perioperative stroke and morbidity rates in the COSS trial. The bypass graft patency rates were excellent (96\% at the last follow-up), and there was significant improvement in the impaired cerebral hemodynamics in the symptomatic cerebral hemisphere on postoperative PET scans in the surgical patients. ${ }^{15} \mathrm{We}$ address the issues raised by Dr. Heros in our response below.
The randomization to 30-day postoperative stroke rates in the 2 randomized trials of EC-IC arterial bypass for stroke prevention are different: $12.2 \%$ for the EC-IC bypass trial ${ }^{9}$ and $15 \%$ for the $\operatorname{COSS}^{15}(p=0.50)$. This $p$ value indicates that one would expect to see a difference as large as the observed difference $50 \%$ of the time if the 2 samples came from a population with the same perioperative stroke risk. However, even if the rate in the COSS had been $12.2 \%$ and the study had been continued to the original sample size of 186 patients in each group, there would not have been a statistically significant difference between the surgical and nonsurgical groups.

It has been suggested that the better-than-expected outcome in the nonsurgical group in the COSS was caused by a failure to use the method of a quantitative oxygen extraction fraction (OEF) threshold of 50\% instead of the COSS ratio method. ${ }^{3}$ The choice of the optimal OEF ratio for the COSS was based on the widely used techniques of receiver operating characteristic curves and cost-effectiveness analysis. ${ }^{6}{ }^{8}$ We have previously shown that the count-based OEF ratio method predicts subsequent stroke as a continuous variable and is superior to the use of an absolute value. ${ }^{7}$ Data from 36 patients in the St. Louis Carotid Occlusion Study (STLCOS) $)^{12}$ demonstrate this. The STLCOS was a prospective blinded observational study of the prognostic value of PET OEF measurements in patients with symptomatic carotid artery occlusion, who met the clinical eligibility criteria for the COSS. We compared quantitative measurements of $\mathrm{OEF}$, done with arterial catheterization for blood measurements, and count-based OEF ratios, calculated identically to those in patients enrolled in the COSS. Of these 36 patients, 9 had subsequent ipsilateral stroke. The 50\% quantitative OEF threshold method only identified 4 of these patients, whereas the COSS ratio method identified 7 , including all 4 identified by the $50 \%$ quantitative threshold method. The risk of subsequent ipsilateral stroke in patients who met the COSS ratio criteria (7 [39\%] of 18) was virtually identical to that in patients with quantitative OEF $\geq 50 \%$ (4 of [40\%] 10). However, since the COSS criteria identified more patients (7 of 9) who would go on to have a stroke than the absolute OEF criteria (4 of 9), it is the superior method for identifying patients with hemodynamic compromise who are at high risk for subsequent stroke. In a recent study, Yamauchi et al. ${ }^{19}$ used a quantitative OEF threshold of 52.9\% to select patients with impaired cerebral hemodynamics. In 16 medically treated patients, the 2-year ipsilateral ischemic stroke rate was $25 \%$ (4 of 16), almost identical to the $22.7 \%$ rate for the nonsurgical group in the COSS..$^{15}$ Thus, there are no data to support the claim that the use of a different PET OEF measurement methodology could identify a high-risk group of patients that would benefit from EC-IC arterial bypass.

The 2-year ipsilateral stroke rate of $22.7 \%$ in the nonsurgical group in the COSS was lower than the $40 \%$ projected rate in the study design. This projected stroke rate of $40 \%$ was derived from a subgroup analysis of the STLCOS. ${ }^{12}$ The hazard ratio for the COSS group as compared with that for the STLCOS subgroup was 0.605 (95\% CI $0.267-1.375, \mathrm{p}=0.231$ ), so the 2 rates are not incompatible. If the true hazards were actually the same in the 2 


\section{Editorial}

populations, one would expect to observe a hazard ratio of this magnitude or smaller $23 \%$ of the time. When we compared 15 available baseline characteristics in the 22 patients in the STLCOS subgroup with those in the 98 nonsurgical patients in the COSS, the only statistically significant differences were related to hyperlipidemia. In the COSS cohort, there was a higher percentage of patients who had a history of hyperlipidemia $(p=0.001)$, who were using any lipid-lowering drugs $(\mathrm{p}=0.001)$, or who were using statins $(p=0.0001)$. In the subgroup of 1007 patients with carotid stenosis in the Stroke Prevention by Aggressive Reduction in Cholesterol Levels (SPARCL) trial, ${ }^{17}$ a difference of $50 \mathrm{mg} / \mathrm{dl}$ in low-density lipoprotein (LDL) cholesterol during the trial reduced the 5-year stroke risk by 33\%. In the COSS, the baseline mean LDL cholesterol level in nonsurgical patients was $54 \mathrm{mg} / \mathrm{dl}$ less than the baseline mean LDL cholesterol level in the STLCOS subgroup, with a similar relative stroke risk reduction of $40 \%$. While a difference in the use of statins is a plausible explanation for the observed difference in the stroke rates in the 2 studies, it remains only a hypothesis. Since the STLCOS did not collect medication data (except for antithrombotic drugs) and did not measure serum lipid levels after baseline, we do not know if the difference in the use of statins or in LDL cholesterol was maintained. Furthermore, this was a comparison of 2 cohorts studied 10 years apart, not a randomized trial, so inferring cause and effect from an observed association is hazardous. Interestingly, a recent trial of endovascular stenting versus aggressive medical therapy for intracranial arterial stenosis also had a much-lower-than-projected stroke rate in the medically treated patients. ${ }^{4}$

It is not valid to assume that the event rates observed during the first 2 years of the COSS would continue and would have led to a significant difference if the study had been continued for 5 years. Such an assumption is inconsistent with other trials of medically treated symptomatic large-artery atherosclerosis that have shown a major decrease in stroke rate after 2 years. In the EC-IC bypass trial, ${ }^{9}$ the stroke rate at 2 years was $20 \%$; at 4 years, the stroke rate had only increased by an additional $6 \%$. In the pooled data from carotid endarterectomy trials, the stroke rate at 2 years for $70 \%-99 \%$ stenosis was $20 \%$, but it only increased by an additional $4 \%$ by Year $4 .{ }^{16} \mathrm{~A} 2 \%-3 \%$ rate of stroke per year in the nonsurgical group in the COSS for an additional 3 years would not have resulted in a statistically significant benefit for surgery, even if no additional strokes occurred in the surgical group.

The COSS was designed to detect a $16 \%$ absolute risk reduction, which was a realistic projection based on data from the STLCOS ${ }^{12}$ and the EC-IC bypass trial. ${ }^{9}$ Final results of the COSS, even with the lower-than-anticipated stroke rate in the nonsurgical group and early termination for futility, excluded with greater than $95 \%$ confidence a benefit this large for surgery. A study of 986 patients with $80 \%$ power to detect a $10 \%$ difference is not feasible and most likely would not have been funded. Based on the $30 \%$ rate of PET scans positive for ipsilateral cerebral ischemia in the COSS, enrollment would have required 3286 patients. This is 4 times the number with symptomatic carotid occlusion enrolled in the EC-IC bypass trial ${ }^{9}$ at 71 international centers over a 5 -year period.
Neither a short time from symptoms to randomization nor recurrent events identified a subgroup at high risk that benefited from surgery in the COSS. Patients in the nonsurgical group were randomized between 5 and 137 days after the qualifying events. An earlier time from the qualifying event to randomization was not a predictor of subsequent stroke. For the subgroup with recurrent events, there was no significant difference in outcome between the surgical and nonsurgical patients. Similar results were observed in the EC-IC bypass trial ${ }^{9}$ in which neither the subgroup with earlier randomization nor the subgroup with recurrent symptoms showed a benefit from surgery. ${ }^{2}$ The COSS was not designed to specifically address the rare patients with postural symptoms and/or an unstable neurological status due to an occluded carotid artery. It is pure speculation that surgical morbidity in these subgroups of unstable patients would be sufficiently low to provide a meaningful clinical benefit. In fact, it is logical to assume that the rate of perioperative morbidity would be even higher than the rates seen in the COSS for EC-IC arterial bypass procedures performed in neurologically unstable patients.

Two well-conducted randomized clinical trials of EC-IC arterial bypass in patients with atherosclerotic cerebrovascular disease have failed to show a benefit for surgery on stroke prevention or functional outcome., ${ }^{9,15}$ The primary criticism of the original EC-IC bypass trial that hemodynamic eligibility criteria were not used was addressed by the COSS. ${ }^{15}$ No clinically important end point has been shown to improve with EC-IC arterial bypass in a randomized controlled trial, and there is currently no method to define a subgroup of patients who benefit from surgery. It is important to remember that the eligibility criteria used in the COSS successfully identified a group of patients with symptomatic carotid artery occlusion who were at very high risk for further stroke with medical therapy. The $22.7 \%$ rate of subsequent ipsilateral stroke is $4-10$ times higher than that for unselected patients with symptomatic carotid artery occlusion and is comparable to that for patients with 70\%-99\% symptomatic carotid artery stenosis. ${ }^{10-16}$

Although a substantially lower perioperative stroke rate in both the initial EC-IC bypass trial ${ }^{9}$ and the COSS trial $^{15}$ might have led to a benefit from the EC-IC arterial bypass operation, it has not been possible to select a group of surgeons within a randomized controlled trial who can operate with a low enough perioperative stroke rate to achieve a benefit for surgery. This is not because of inexperienced surgeons and poor surgical techniques in previous clinical trials, but because patients with carotid artery occlusion and impaired cerebral hemodynamics have a significant risk for perioperative stroke and morbidity due to compromised brain function and the existence of other significant comorbidities.

\section{References}

1. Ausman JI, Diaz FG: Critique of the extracranial-intracranial bypass study. Surg Neurol 26:218-221, 1986

2. Barnett HJ, Fox A, Hachinski V, Haynes B, Peerless SJ, Sackett $\mathrm{D}$, et al: Further conclusions from the extracranial-intracranial bypass trial. Surg Neurol 26:227-235, 1986 
3. Carlson AP, Yonas H, Chang YF, Nemoto EM: Failure of cerebral hemodynamic selection in general or of specific positron emission tomography methodology? Carotid Occlusion Surgery Study (COSS). Stroke 42:3637-3639, 2011

4. Chimowitz MI, Lynn MJ, Derdeyn CP, Turan TN, Fiorella D, Lane BF, et al: Stenting versus aggressive medical therapy for intracranial arterial stenosis. N Engl J Med 365:993-1003, 2011 (Erratum in N Engl J Med 367:93, 2012)

5. Day AL, Rhoton AL Jr, Little JR: The extracranial-intracranial bypass study. Surg Neurol 26:222-226, 1986

6. Derdeyn CP, Gage BF, Grubb RL Jr, Powers WJ: Cost-effectiveness analysis of therapy for symptomatic carotid occlusion: PET screening before selective extracranial-to-intracranial bypass versus medical treatment. J Nucl Med 41:800-807, 2000

7. Derdeyn CP, Videen TO, Grubb RL Jr, Powers WJ: Comparison of PET oxygen extraction fraction methods for the prediction of stroke risk. J Nucl Med 42:1195-1197, 2001

8. Derdeyn CP, Videen TO, Simmons NR, Yundt KD, Fritsch SM, Grubb RL Jr, et al: Count-based PET method for predicting ischemic stroke in patients with symptomatic carotid arterial occlusion. Radiology 212:499-506, 1999

9. EC-IC Bypass Study Group: Failure of extracranial-intracranial arterial bypass to reduce the risk of ischemic stroke. Results of an international randomized trial. N Engl J Med 313:11911200,1985

10. Flaherty ML, Flemming KD, McClelland R, Jorgensen NW, Brown RD Jr: Population-based study of symptomatic internal carotid artery occlusion: incidence and long-term follow-up. Stroke 35:e349-e352, 2004

11. Goldring S, Zervas N, Langfitt T: The Extracranial-Intracranial Bypass Study. A report of the committee by the American Association of Neurological Surgeons to examine the study. $\mathbf{N}$ Engl J Med 316:817-820, 1987

12. Grubb RL Jr, Derdeyn CP, Fritsch SM, Carpenter DA, Yundt
$\mathrm{KD}$, Videen TO, et al: Importance of hemodynamic factors in the prognosis of symptomatic carotid occlusion. JAMA 280: 1055-1060, 1998

13. Hankey GJ, Warlow CP: Prognosis of symptomatic carotid artery occlusion. Cerebrovasc Dis 1:245-256, 1991

14. Klijn CJM, Kappelle LJ, Tulleken CAF, van Gijn J: Symptomatic carotid artery occlusion. A reappraisal of hemodynamic factors. Stroke 28:2084-2093, 1997

15. Powers WJ, Clarke WR, Grubb RL Jr, Videen TO, Adams HP Jr, Derdeyn CP: Extracranial-intracranial bypass surgery for stroke prevention in hemodynamic cerebral ischemia: the Carotid Occlusion Surgery Study randomized trial. JAMA 306: 1983-1992, 2011 (Erratum in JAMA 306:2672, 2011)

16. Rothwell PM, Eliasziw M, Gutnikov SA, Fox AJ, Taylor DW, Mayberg MR, et al: Analysis of pooled data from the randomised controlled trials of endarterectomy for symptomatic carotid stenosis. Lancet 361:107-116, 2003

17. Sillesen H, Amarenco P, Hennerici MG, Callahan A, Goldstein LB, Zivin J, et al: Atorvastatin reduces the risk of cardiovascular events in patients with carotid atherosclerosis: a secondary analysis of the Stroke Prevention by Aggressive Reduction in Cholesterol Levels (SPARCL) trial. Stroke 39:3297-3302, 2008

18. Sundt TM Jr: Was the international randomized trial of extracranial-intracranial arterial bypass representative of the population at risk? N Engl J Med 316:814-816, 1987

19. Yamauchi H, Higashi T, Kagawa S, Nishii R, Kudo T, Sugimoto $\mathrm{K}$, et al: Is misery perfusion still a predictor of stroke in symptomatic major cerebral artery disease? Brain 135:2515-2526, 2012

Please include this information when citing this paper: published online October 26, 2012; DOI: 10.3171/2012.6.JNS121106. 